\title{
Identification of Pns12 as the second silencing suppressor of Rice gall dwarf virus
}

\author{
WU JianGuo ${ }^{1,2}$, WANG ChunZheng ${ }^{2}$, DU ZhengGuo ${ }^{1}$, CAI LiJun ${ }^{1}$, HU MeiQun ${ }^{1}$, \\ WU ZuJian ${ }^{1 *}$, LI Yi $^{2^{*}} \&$ XIE LianHui ${ }^{*}$ \\ ${ }^{1}$ Institute of Plant Virology, Fujian Agriculture and Forestry University, Key Laboratory of Plant Virology of Fujian Province, \\ Fuzhou 350002, China; \\ ${ }^{2}$ Peking-Yale Joint Center for Plant Molecular Genetics and Agrobiotechnology, National Laboratory of Protein \\ Engineering and Plant Genetic Engineering, College of Life Sciences, Peking University, Beijing 100871, China
}

\begin{abstract}
RNA silencing is a conserved mechanism found ubiquitously in eukaryotic organisms. It has been used to regulate gene expression and development. In addition, RNA silencing serves as an important mechanism in plants' defense against invasive nucleic acids, such as viruses, transposons, and transgenes. As a counter-defense, most plants, and some animal viruses, encode RNA silencing suppressors to interfere at one or several points of the silencing pathway. In this study, we showed that Pns12 of RGDV (Rice gall dwarf virus) exhibits silencing suppressor activity on the reporter green fluorescent protein in transgenic Nicotiana benthamiana line 16c. Pns12 of RGDV suppressed local silencing induced by sense RNA but had no effect on that induced by dsRNA. Expression of Pns12 also enhanced Potato virus $X$ pathogenicity in N. benthamiana. Collectively, these results suggested that RGDV Pns12 functions as a virus suppressor of RNA silencing, which might target an upstream step of dsRNA formation in the RNA silencing pathway. Furthermore, we showed that Pns12 is localized mainly in the nucleus of $N$. benthamiana leaf cells.
\end{abstract}

\section{Rice gall dwarf virus, Pns12, silencing suppressor}

Citation: Wu J G, Wang C Z, Du Z G, et al. Identification of Pns12 as the second silencing suppressor of Rice gall dwarf virus. Sci China Life Sci, 2011, 54: 201-208, doi: 10.1007/s11427-011-4142-5

RNA silencing is a conserved mechanism used by plants and other eukaryotes to defend themselves against viruses and transposons [1]. Generally, RNA silencing is triggered by dsRNAs. The dsRNAs are recognized by specific cellular enzymes and cleaved into small interfering RNAs (siRNAs) of 20-24 nt in length. The siRNAs function as guides for a silencing complex that can block gene expression at transcriptional, post-transcriptional, and translational levels [2-6]. Most viruses produce dsRNAs during their life cycles. This means that viruses can be both triggers and targets of RNA silencing [7,8]. As a counter-defense, most

*Corresponding author (email: wuzujian@126.com; liyi@pku.edu.cn; fjxlh@126.com) plant viruses have evolved to encode a protein that can suppress RNA silencing [1,9-13].

A number of studies have shown that VSRs (viral suppressors of RNA silencing) target distinct steps of RNA silencing: from the production of dsRNAs to the incorporation of siRNAs into the RNA-induced silencing complex (RISC) $[1,14,15]$. In addition, some VSRs have been shown to interfere with the movement of silencing signals. A number of VSRs have been identified from plant and animal viruses [15-25]. Although most plant viruses only have one VSR, some plant viruses encode more than one silencing suppressor, which target distinct steps of RNA silencing [26,27].

Rice gall dwarf virus (RGDV) is a member of the genus 
Phytoreovirus under the family Reoviridae. Besides RGDV, viruses of the genus Phytoreovirus include Rice dwarf virus, Wound tumor virus [28], Rice bunchy stunt virus [29], Tobacco leaf enation virus [30] and Homalodisca vitripennis virus (HVP) [31]. RGDV has a double-shelled icosahedral particle. Its genome consists of 12 double-stranded RNAs, which encode six structural (P1, P2, P3, P5, P6, and P8) and six non-structural (Pns4, Pns7, Pns9, Pns10, Pns11, and Pns12) proteins. The core capsid is composed of P3, the major structural protein, which encloses P1, P5, and P6. The outer layer consists of two proteins, P2 and P8 [32]. RGDV is transmitted by Recilia dorsalis and Nephotettix cincticeps in a persistent and circulative manner [33]. The complete sequences of RGDV Thai [34,35] and Guangxi [36,37] isolates have been obtained, which paved the way for molecular studies on the functions of RGDV gene products and the RGDV-rice interaction.

S12, the smallest RNA segment of RGDV, is $853 \mathrm{bp}$ long. It contains an ORF of $620 \mathrm{bp}$, from 31 to $650 \mathrm{nt}$ [38]. To date, the function of the protein encoded by S12 and Pns12 remains unknown. A previous report showed that RGDV S11 encodes a silencing suppressor [21]. In this study, we present evidence that Pns12 of RGDV also has silencing suppressor activities. Pns12 of RGDV exhibited silencing suppressor activity in coinfiltration assays with the reporter green fluorescent protein (GFP) in transgenic Nicotiana benthamiana line 16c. It suppressed local silencing induced by sense RNA, but had no effect on that induced by dsRNA. Expression of Pns12 also enhanced Potato virus $X$ pathogenicity in $N$. benthamiana. In addition, we showed that Pns12 localizes mainly to the nucleus in $N$. benthamiana cells, which suggested that Pns12 might function in the nucleus.

\section{Materials and methods}

\subsection{Plasmids and Agrobacterium}

Rice plants infected by RGDV, which were collected from Xinyi, Guangdong Province, were used as the source of RGDV. The viruses were transmitted artificially using its insect vectors to rice seedlings and the seedlings were grown and maintained in the greenhouse of the Institute of Plant Virology, Fujian Agriculture and Forestry University.

The primers for S12, 5'-CGCAAGCTTATGACGAGCAACGAGGAAAAC-3' and 5'-GAGGAGCTCTTACCTCGGTCTTCGTTTAC-3', were designed according to sequences in NCBI (GenBank accession No. EF177263; restriction enzyme cleavage sites are underlined). The full open reading frame of Pns12 was amplified from pMD-18T vector containing RGDV S12. The PCR products were cleaved using Hind III and $S a C$ I and then transferred into the binary vector pPZP212 [39] (a kind gift from Dr. Powers), creating pPZP212-S12 (named 35S:S12 for simplicity).
The primer 5'-CGCAAGCTTTGACGAGCAACGAGGAAAAC-3' was used to obtain $\triangle \mathrm{S} 12$. PCR amplification using this primer results in the deletion of the first nucleic acid of the S12 ORF. $\triangle \mathrm{S} 12$ was transferred into the same binary vector as $\mathrm{S} 12$, resulting in pPZP212- $\Delta \mathrm{S} 12$ (named $35 \mathrm{~S}: \Delta \mathrm{S} 12$ for simplicity). Deletion of the first nucleic acid of the S12 ORF leads to a construct that can be transcribed but cannot be translated into a protein. Similarly, the primer 5'-ATACCCGGGTGACGAGCAACGAGGAAAAC-3' was used to create PVX: $\triangle \mathrm{S} 12$ (see below).

The ORF of $\mathrm{S} 12$ was amplified using primers $5^{\prime}$-AT-

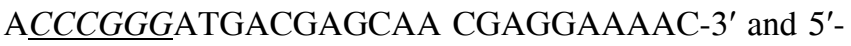
ACGGTCGACTTACCTCGGTCTTCGTTTAC-3', which contains recognition sites for the enzymes Sma I and Sal I. The PCR products were ligated into the vector pGR107 (a kind gift from David Baulcombe) after double digestion using Sma I and Sal I. The recombinant plasmids were named PVX:S12. PVX: $\triangle \mathrm{S} 12$ was obtained by using the primer 5'-ATA $\underline{C C C G G T G A C G A G C A A C G A G G A A A A-}$ C-3'.

The S12 ORF lacking the stop codon was obtained using the primers 5'-ATA $\underline{C A T G G A A A T G A C G A G C A A C G A G-}$ GAAA-3' and 5'-ATA $\underline{C A T G G A C C T C G G T C T T C G T T T-}$ ACTTT-3', which contained the recognition sites for the enzyme Nco I. The PCR products were ligated into the vector pRTL2-GFP, resulting in pRTL2-S12:GFP, which, after digestion with the enzyme Hind III, was transferred into the binary vector pCAMBIA1301, creating pCAMBIA1301S12:GFP (35S:S12:GFP for simplicity).

The constructs pPZP212, 35S:S12, 35S: $\triangle \mathrm{S} 12$, 35S:ssGFP, 35S:dsGFP, 35S:TAV 2b, PVX, PVX:S12, PVX: $\triangle S 12$, and $35 \mathrm{~S}: \mathrm{S} 12$ :GFP were sequenced to verify their correct construction and transformed into Agrobacterium tumefaciens GV3101 (kindly provided by David Baulcombe) by electroporation.

\subsection{Agroinfiltration and GFP imaging}

The Nicotiana benthamiana plant constitutively expressing a GFP transgene (line 16c; a gift from David Baulcombe) and the Agrobacterium infiltration operation have been described previously [40]. The $N$. benthamiana line $16 \mathrm{c}$ plants were cultured in growth chambers at $22-24^{\circ} \mathrm{C}$ before and after infiltration. GFP fluorescence was observed under long wavelength UV light (Black Ray model B 100A; UV Products) and photographed using a Nikon D70 digital camera with a Y48 yellow filter.

\subsection{RNA extraction and Northern blotting}

Total RNAs were extracted from leaves with TRIzol reagent (Invitrogen) in accord with the manufacturer's instructions. Northern blotting analysis was conducted according to instructions described in "the DIG system user's guide for 
filter hybridization". For Northern blot analysis of siRNAs, low-molecular-weight RNAs were enriched from total RNAs by eliminating high-molecular-weight RNA using $5 \%$ polyethylene glycol (PEG 8000 ) plus $0.5 \mathrm{~mol} \mathrm{~L}^{-1} \mathrm{NaCl}$, separating on a $15 \%$ polyacrylamide $-7 \mathrm{~mol} \mathrm{~L}^{-1}$ urea gel, and transferring to Hybond-N membranes. The hybridization and detection of siRNAs were performed as described previously [41]. The probes used in the analysis of siRNA were the same as those for Northern blots of mRNA.

\subsection{Localization of Pns12}

The leaves of four-week-old $N$. benthamiana plants were agroinfiltrated with A. tumefaciens harboring pCAMBIA1301-S12:GFP and pCAMBIA1301-GFP, respectively. By 3 dpi (days postinfiltration), the GFP expression in the leaves was observed at $488 \mathrm{~nm}$ using a confocal microscope (Leica TCS STED, Germany) and DAPI (2-(4-Amidinophenyl)-6-indolecarbamidine dihydrochloride) to counterstain the nuclei. Images were captured using LAS AF Lite software and converted to tagged image file format for export.

\section{Results}

\subsection{RGDV Pns12 inhibits local silencing induced by ssGFP}

To determine whether RGDV Pns12 has silencing suppressor activities, a method named the agroinfiltration bioassay was used. A transformed Agrobacterium strain carrying RGDV Pns 12 was mixed with a strain that carried 35S-GFP at a ratio of 3:1 and infiltrated into leaves of $N$. benthamiana line 16c. Agrobacteria harboring only the GFP gene or the $2 \mathrm{~b}$ gene of Tomato aspermy cucumovirus (TAV) was used as negative and positive controls, respectively. In each treatment, 15 plants were infiltrated and the experiments were repeated at least three times. GFP fluorescence became visible at 2 dpi in localized regions. However, the green fluorescence intensity in the patches infiltrated with GFP alone or with GFP plus $\Delta \mathrm{S} 12$, in which the first nucleic acid of the S12 ORF was deleted, became invisible after 7 dpi. By contrast, the green fluorescence intensity remained strong at 7 dpi in the patches coinfiltrated with $35 \mathrm{~S}-\mathrm{GFP}$ plus $35 \mathrm{~S}-\mathrm{S} 12$ and 35S-GFP plus 35S-TAV 2b, respectively (Figure 1A-F). These results suggest that Pns12 could suppress the RNA silencing induced by sense-GFP, presumably in a similar manner to TAV $2 b$. In addition, the fact that $\Delta S 12$, which could not produce an intact Pns12, had no effect on RNA silencing indicated it was the protein expressed from $\mathrm{S} 12$, but not the RNA, that could function as a silencing suppressor.

To confirm that the above observations resulted from differential accumulation of GFP mRNA, Northern blot analyses were conducted to detect steady-state levels of GFP mRNA using digoxin labeled probes. The results showed that GFP mRNA accumulated in tissues expressing 35S:GFP plus 35S:TAV 2b or 35S:GFP plus 35S:S12 and were high at $7 \mathrm{dpi}$, although the accumulated levels of GFP mRNA in tissues expressing 35S:GFP plus 35S:S12 were much lower than in tissues expressing 35S:GFP plus 35S:TAV 2b. By contrast, GFP mRNA could not be detected in tissues expressing GFP alone or 35S:GFP plus $35 \mathrm{~S}: \Delta \mathrm{S} 12$ at the same time point (Figure 1G).

To further test whether RNA silencing suppressor activities of PnS12 were responsible for the above observations, Northern blot analyses were conducted to detect GFP-specific siRNAs. In all treatments, higher accumulation of GFP mRNA was correlated to lower accumulation of GFP-specific siRNAs, and lower accumulation of GFP mRNA was accompanied by higher accumulation of GFP-specific siRNAs (Figure 1G). These results indicated that transcription of the exogenously introduced sense-GFP can induce co-suppression or RNA silencing of both the exogenous and the endogenous GFP. RGDV PnS12 can suppress the RNA silencing induced by sense-GFP, in a similar manner to TAV 2b, although its silencing suppressor activities were much weaker than the latter.

\subsection{Pns12 does not suppress local RNA silencing trig- gered by GFP dsRNA}

DsRNA, as found by Fire et al. [7] in 1998, is the initiator of RNA silencing. RNA silencing involves three distinct stages: the generation of dsRNA, the cleavage of the dsRNAs into siRNAs 20-26 nt in length, and the incorporation of the siRNAs into an effector complex $[12,42]$. Many studies have used dsGFP in co-infiltration assays to determine the molecular targets of a particular VSR. It was found that VSRs, including Pns10 of Rice dwarf virus [20], P69 of Turnip yellow mosaic virus (TYMV) [43], and P25 of Potato virus $X$ (PVX) [44] could not inhibit RNA silencing induced by dsRNAs, suggesting that these VSRs targeted a step upstream of dsRNA formation in RNA silencing. Our results indicated that Pns 12 could inhibit local silencing of GFP triggered by ssGFP. However, the targets of Pns12 in the RNA silencing were unknown.

The effect of Pns12 on dsGFP-triggered silencing was tested, using 35S:TAV 2b plus 35S:ssGFP plus 35S:dsGFP and $35 \mathrm{~S}:$ ssGFP plus $35 \mathrm{~S}: \mathrm{dsGFP}$ plus $35 \mathrm{~S}: \Delta \mathrm{S} 12$ or the empty vector as positive and negative controls, respectively. As shown in Figure 2, leaves infiltrated with 35S-ssGFP plus $35 \mathrm{~S}$-dsGFP or with $35 \mathrm{~S}$-ssGFP plus $35 \mathrm{~S}$-dsGFP plus $35 \mathrm{~S}-\mathrm{S} 12$ (or $35 \mathrm{~S}-\Delta \mathrm{S} 12$ ) lost GFP fluorescence at $7 \mathrm{dpi}$, indicating strong local GFP RNA silencing. This indicated that Pns12 could not suppress local silencing induced by dsRNA. As expected, strong GFP fluorescence was main- 

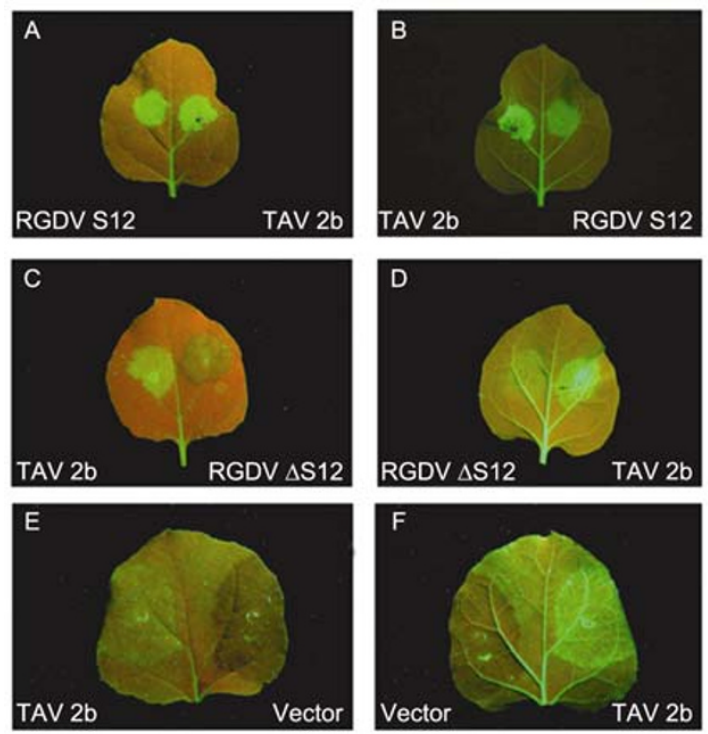

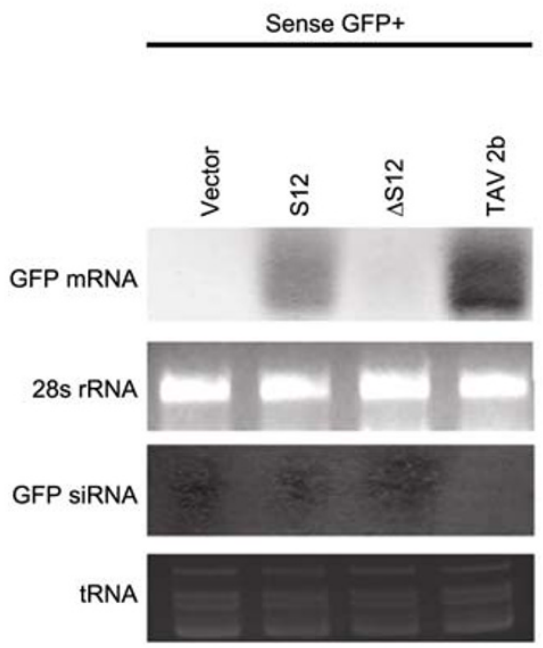

Figure 1 Suppression of local GFP silencing by RGDV Pns12 (A-F). N. benthamiana line 16c plants were coinfiltrated with Agrobacterium spp. (Agro.) mixtures carrying 35S-GFP and the individual constructs indicated in each image. GFP fluorescence was viewed under long wavelength UV light at $7 \mathrm{~d}$ postinfiltration (dpi). G, Northern blot analysis of the steady-state levels of GFP mRNA and siRNA extracted from different infiltrated patches shown in A-F. rRNA and tRNA were used as loading controls for detection of GFP mRNA and GFP siRNA, respectively.
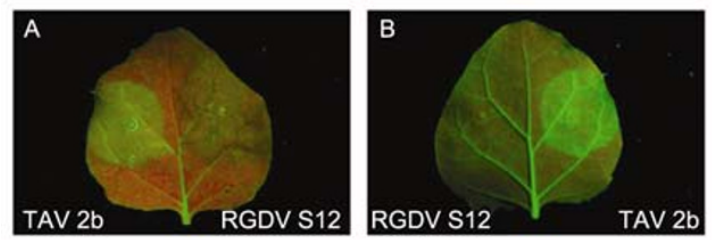

G
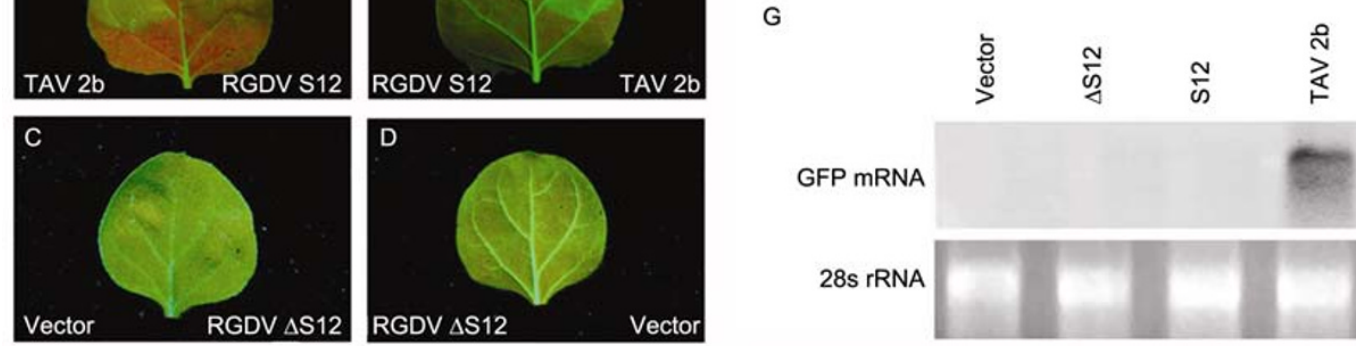

GFP mRNA
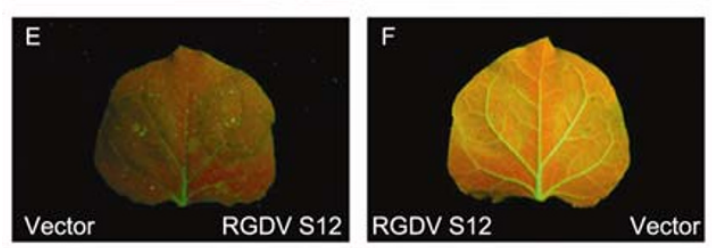

28s rRNA

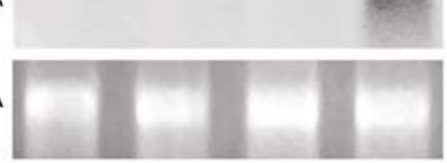

Figure 2 RGDV Pns12 did not inhibit local silencing induced by dsRNA (A-F). N. benthamiana line 16c plants were coinfiltrated with Agrobacterium spp. (Agro.) mixtures carrying 35S:dsGFP and the individual constructs indicated in each image. GFP fluorescence was viewed under long wavelength UV light at $7 \mathrm{~d}$ postinfiltration (dpi). G, Northern blot analysis of the steady-state levels of GFP mRNA extracted from different infiltrated patches shown in A-F. The bottom gel shows 28 s rRNA with ethidium bromide staining as a loading control.

tained in leaves infiltrated with 35S-ssGFP plus 35S-dsGFP plus 35S:TAV 2b, indicating that TAV 2b suppressed local GFP RNA silencing triggered by dsRNA (Figure 2A-F). Northern blot analyses showed negligible accumulation of GFP mRNA and high accumulation of GFP-specific siRNAs in leaves infiltrated with 35S-ssGFP plus 35S-dsGFP and 35SssGFP plus 35S:dsGFP plus 35S:S12/35S: $\Delta$ S12 (Figure $2 \mathrm{G}$ ), further demonstrating that Pns12 did not suppress local RNA silencing triggered by GFP dsRNA. By contrast, leaves infiltrated with 35S:ssGFP plus 35S:dsGFP plus 35S:TAV $2 b$ showed high accumulation of GFP mRNA and much reduced accumulation of siRNA (Figure 2G). These results suggested that Pns12 targets a step upstream of dsRNA synthesis in the RNA silencing pathway.

\subsection{Pns12 enhances PVX pathogenicity}

Studies of Voinnet et al. [44] suggested that the RNA si- 
lencing triggered by ssRNA and viruses might involve different mechanisms. P25 of PVX can inhibit ssRNA induced silencing. However, it had no apparent effects on silencing induced by viruses [44]. Synergism refers to a phenomenon in which plants infected with two unrelated viruses displayed increased disease symptoms compared with plants infected with either of the two viruses alone [45]. Currently, it is generally accepted that synergism is the result of very effective suppression of silencing by one virus, resulting in a dramatic increase in the accumulation of the co-infecting virus [46]. The above results showed that PnS12 of RGDV can suppress local silencing induced by ssRNA when transiently expressed in $N$. benthamiana. However, the function of the silencing suppressor activity of PnS12 in natural viral infection is unknown. As a dsRNA virus with a large genome, no infectious clones for reverse genetic studies of RGDV were available, making a direct test of the role of PnS12 in RGDV infection of rice impossible.

To study the role of Pns12 in viral infection in the context of plant-virus interaction, we utilized a PVX vector to express S12 and an ORF frame-shift mutant $(\Delta S 12)$. Seedlings of $N$. benthamiana plants (four- to six-leaf stage) were inoculated with PVX, PVX-S12, and PVX- $\Delta \mathrm{S} 12$, respectively. All inoculated leaves were asymptomatic. However, symptoms were visible in systemically infected leaves inoculated with PVX or PVX- $\Delta \mathrm{S} 12$ as early as 6 dpi. Generally, the symptoms caused by PVX or PVX- $\Delta \mathrm{S} 12$ developed initially as veinal chlorosis between 6 and 9 dpi but subsequently (9-18 dpi) as mild chlorotic spots in some leaves. In addition, some newly developed leaves became asymptomatic, a phenomenon reminiscent of recovery from viral infection. The symptoms caused by PVX-S12 developed one to two days later than those caused by PVX or PVX- $\Delta$ S12. However, the symptoms caused by PVX-S12 became indistinguishable from those caused by PVX or PVX- $\Delta$ S12 at 9 dpi. At $12 \mathrm{dpi}$, it became evident that the symptoms caused by PVX-S12 were more severe than those caused by PVX or PVX- $\Delta \mathrm{S} 12$. This became even more obvious at $18 \mathrm{dpi}$. At this time, symptoms caused by PVX-S12 were still visible, whereas only very slight symptoms could be observed in some of the leaves of PVX or PVX- $\Delta \mathrm{S} 12$ inoculated plants (Figure 3A).

To investigate the mechanisms by which Pns 12 caused increased disease symptoms in the heterologous system, Northern blots were carried out to detect the accumulated levels of the genomic RNAs of PVX. As shown in Figure 3B, PVX mRNAs accumulated to high levels in systemic leaves of $N$. benthamiana infected by either PVX, PVX: $\triangle \mathrm{S} 12$ or PVX-S12 at 6 dpi. However, the accumulation of PVX mRNAs decreased significantly in plants infected by either PVX or PVX: $\triangle \mathrm{S} 12$ at $18 \mathrm{dpi}$. By contrast, the accumulated level of PVX mRNAs remained very high in plants infected by PVX-S12 at this time. Taken together, these results indicated that the presence of Pns12 resulted in increased disease symptoms in PVX infected $N$. benthamiana. The in- creased disease symptoms were probably a result of enhanced replication of PVX, which in turn might be caused by the suppression of RNA silencing by Pns 12 .

\subsection{Cellular localization of RGDV Pns12}

To determine the subcellular localization of Pns12, the S12 coding sequence was fused in-frame to the 5 terminus of the GFP gene driven by a Cauliflower mosaic virus 35S promoter. The constructs were introduced into $N$. benthamiana leaf cells via agroinfiltration. GFP fluorescence was observed using confocal microscopy 3 dpi. As shown in Figure 4, the fluorescence occurred in the nuclei of leaves expressing the S12:GFP fusion protein. However, an examination of leaves expressing free GFP by confocal microscopy showed fluorescence distributed evenly in the cytoplasm and nuclei. The nuclear localization of Pns12 was consistent with our predications based on sequence analysis using PSORT II Prediction (http://psort.ims.u-to-kyo.ac.jp/ form2.html), which showed that a region located in the $\mathrm{C}$ terminus of Pns12 was rich in basic amino acids and might function as a nuclear localization signal (NLS). The relationship between the nuclear localization and the silencing suppressor activities of Pns12 warrants further investigation.

\section{Discussion}

Recent studies have shown that a specific plant virus can encode more than one silencing suppressors. For example, Citrus tristeza virus (CTV) encodes three VSRs. The three VSRs, namely p20, p23, and CP, can function independently at the intracellular or intercellular levels [4,26]. In addition, Cui et al. [47] demonstrated that a Geminivirus, Tobacco curly shoot virus Y35 (TbCSV-Y35), could process three VSRs, namely AC2, AC4, and $\beta \mathrm{C} 1$. Liu et al. [21] have reported that RGDV Pns11 was a silencing suppressor. Here, we showed that Pns12 might be another silencing suppressor of RGDV. Pns12 could suppress silencing induced by sense-GFP. It can reduce, but not eliminate, the accumulation of siRNAs arising from local silencing of GFP. These results indicated that Pns12 might target an initial step of RNA silencing, having a similar mode of action to 2b of CMV [48] and p69 of TYMV [43]. Supporting this, we showed that Pns12 could not inhibit silencing induced by GFP dsRNA (Figure 2). In addition, Pns12 could enhance the pathogenicity of PVX in a heterologous system. The introduction of Pns12 into the genome of PVX resulted in increased viral accumulation in infected $N$. benthamiana (Figure 3B). This suggested that Pns12 might have an important role in RGDV infection of rice.

Our observation indicates that the silencing suppressor activities of Pns12 are weaker than those of Pns11. Both Pns12 and Pns11 can inhibit ssRNA-induced silencing and 

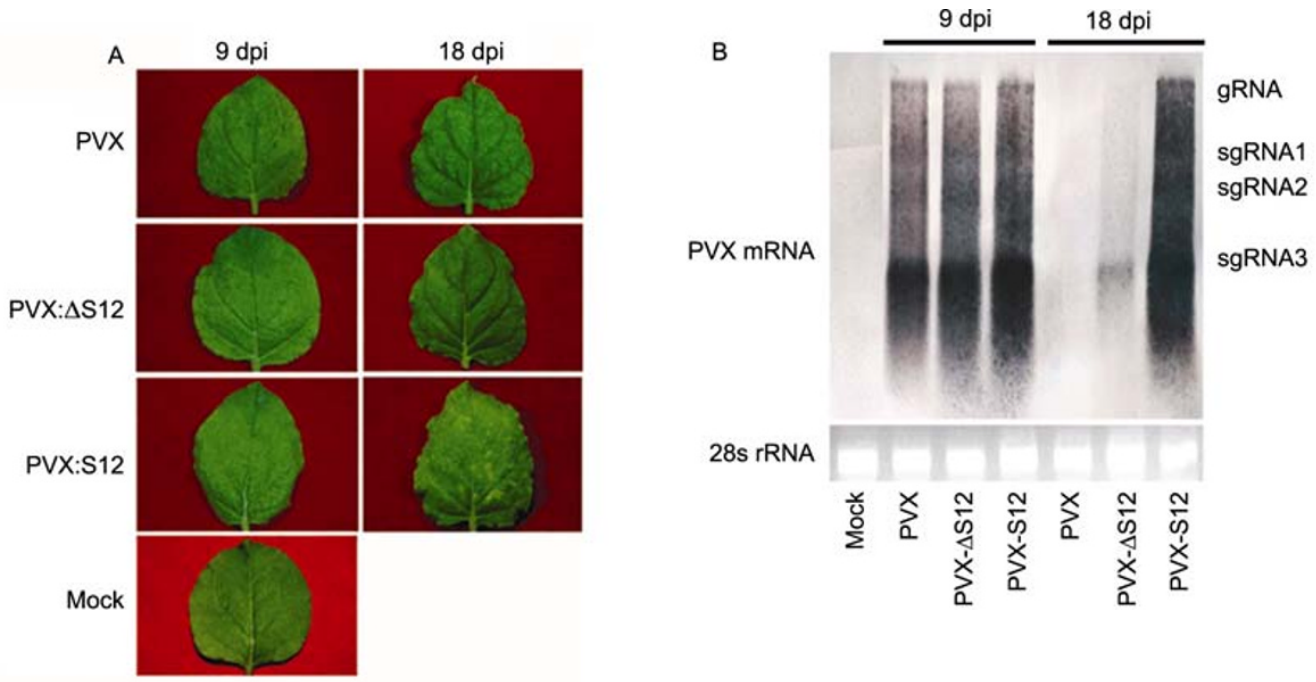

Figure 3 RGDV Pns12 enhances pathogenicity of chimeric PVX. A, Plants infected by PVX or PVX: $\triangle \mathrm{S} 12$ show mild disease symptoms as a few scattered chlorotic speckles, whereas leaves infected with PVX:S12 show more severe symptoms. B, RNA gel blot analysis of accumulation of PVX genomic (gRNA) and subgenomic mRNAs (sgRNA1 to sgRNA3) at 9 and 18 dpi. The bottom gel shows rRNA with ethidium bromide staining as a loading control.
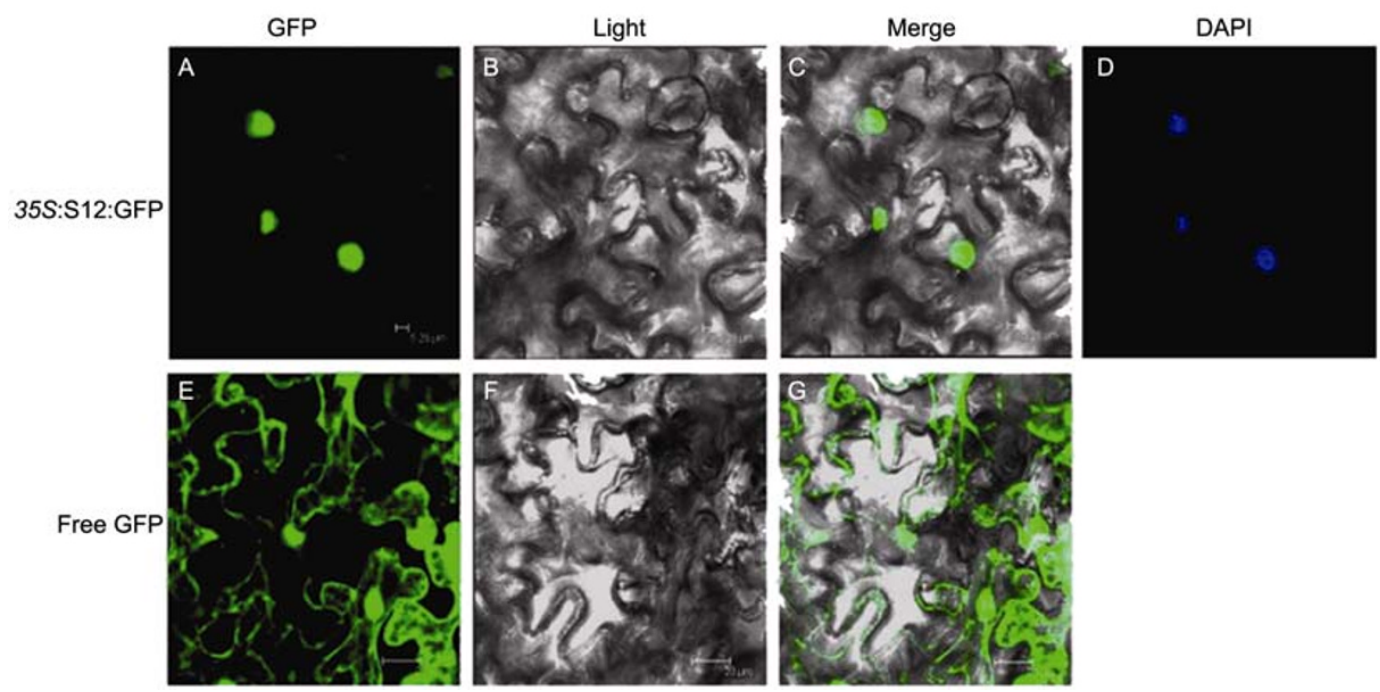

Figure 4 Subcellular localization of 35S:S12:GFP fusion protein transiently expressed in tobacco epidermis cells. A, Fluorescence from the Pns12 tagged with GFP accumulated in the nucleus. B, The same cell as in A under bright-field illumination (C) is an overlay of images A and B. D, DAPI stained nucleus. E, Accumulation of GFP in the cytoplasm and nucleus. F, The same cell as in E under bright-field illumination. G, An overlay of bright and fluorescent illumination of $\mathrm{E}$ and $\mathrm{F}$.

enhance the pathogenicity of PVX in a heterologous system. However, unlike Pns11, Pns12 could not suppress systemic silencing triggered by ssRNAs (data not shown). However, the effects of Pns 11 on silencing induced by dsRNAs are unknown at present. Thus, we suggest that Pns12 and Pns11 target different steps in the RNA silencing pathway.

Transient expression of Pns12 in N. benthamiana showed that this protein mainly accumulated in the nucleus. Nuclear localization is essential for the silencing suppressor activities of some VSRs. For example, the nuclear localization of the protein 2b, a VSR of Cucumber mosaic virus (CMV), was closely related to its pathogenicity [49]. Studies on $2 b$ indicated that it could interact with AGO1, a major protein of the RNA-induced silencing complex (RISC). The interaction resulted in the suppression of the slicer activity of the RISC and thus compromised the miRNA pathway [50]. Nuclear accumulation was also required for these activities of $2 b$ [51]. Nuclear accumulation was also required for the silencing suppressor activity and induction of necrosis of Tomato yellow leaf curl virus-China (TYLCV-C) C2 [52,53]. In addition, a recent study of Xiong et al. [23] showed that NS3, the VSR of Rice stripe virus (RSV) localized to the nuclei. Deletion of the NSL of NS3 resulted in reduced silencing suppressor activity. We hypothesize that Pns12 enters the nucleus and inactivates certain protein components of the RNA silencing pathway through physical interactions 
with them. However, further studies are required to correlate the nuclear accumulation of Pns12 with its silencing suppressor activities. Additionally, it will be interesting to further characterize the role of Pns12 in RGDV infection of rice.

This work was supported by the National Basic Research Program of China (Grant No. 2010CB126203), the National Transgenic Major Program (Grant Nos. 2009ZX08009-044B and 2009ZX08001-018B), the National Natural Science Foundation of China (Grant No. 30970135), the Fujian Province Education Department (Grant No. JB08078), and Specialized Research Fund for the Ministry of Agriculture (Grant No. nyhyzx 07-051).

1 Ding S W, Voinnet O. Antiviral immunity directed by small RNAs. Cell, 2007, 130: 413-426

2 Fire A. RNA-triggered gene silencing. Trends Genet, 1999, 15: 358-363

3 Caplen N J, Fleenor J, Fire A, et al. dsRNA-mediated gene silencing in cultured Drosophila cells: A tissue culture model for the analysis of RNA interference. Gene, 2005, 252: 95-105

4 Caplen N J, Parrish S, Imani F, et al. Specific inhibition of gene expression by small double-stranded RNAs in invertebrate and vertebrate systems. Proc Natl Acad Sci USA, 2001, 98: 9742-9747

5 Hammond S M, Bernstein E, Beach D, et al. An RNA-directed nuclease mediates post-transcriptional gene silencing in Drosophila cells. Nature, 2000, 404: 293-296

6 Elbashir S M, Lendeckel W, Tuschl T. RNA interference is mediated by 21- and 22-nucleotide RNAs. Gene Dev, 2001, 15: 188-200

7 Fire A, Xu S Q, Montgomery M K, et al. Potent and specific genetic interference by double-stranded RNA in Caenorhabditis elegans. Nature, 1998, 391: 806-811

8 Zamore P D. Ancient pathways programmed by small RNAs. Science, 2002, 296: 1265-1269

9 Ding S W. RNA silencing. Curr Opin Biotech, 2000, 11: 152-156

10 Vance V, Vaucheret H. RNA silencing in plants-defense and counterdefense. Science, 2001, 292: 2277-2280

11 Ding S W, Li H W, Lu R, et al. RNA silencing: A conserved antiviral immunity of plants and animals. Virus Res, 2004, 102: 109-115

12 Roth B M, Pruss G J, Vance V B. Plant viral suppressors of RNA silencing. Virus Res, 2004, 102: 97-108

13 Voinnet O. Induction and suppression of RNA silencing: Insights from viral infections. Nat Rev Genet, 2005, 6: 206-220

14 Qi Y J, Zhong X H, Itaya A, et al. Dissecting RNA silencing in protoplasts uncovers novel effects of viral suppressors on the silencing pathway at the cellular level. Nucleic Acids Res, 2004, 32: e179

15 Li F, Ding S W. Virus counterdefense: Diverse strategies for evading the RNA-silencing immunity. Annu Rev Microbiol, 2006, 60: 503-531

16 Anandalakshmi R, Pruss G J, Ge X, et al. A viral suppressor of gene silencing in plants. Proc Natl Acad Sci USA, 1998, 95: 13079-13084

17 Brigneti G, Voinnet $\mathrm{O}, \mathrm{Li} \mathrm{W}$ X, et al. Viral pathogenicity determinants are suppressors of transgene silencing in Nicotiana benthamiana. EMBO J, 1998, 17: 6739-6746

18 Kasschau K D, Carrington J C. A counterdefensive strategy of plant viruses: Suppression of posttranscriptional gene silencing. Cell, 1998, 95: 461-470

19 Lecellier C H, Dunoyer P, Arar K, et al. A cellular microRNA mediates antiviral defense in human cells. Science, 2005, 308: 557-560

20 Cao X S, Zhou P, Zhang X M, et al. Identification of an RNA silencing suppressor from a plant double-stranded RNA virus. J Virol, 2005, 79: 13018-13027

21 Liu F, Zhao Q, Ruan X, et al. Suppressor of RNA silencing encoded by Rice gall dwarf virus genome segment 11. Chin Sci Bull, 2008, 53: 96-103

22 Schnettler E, Hemmes H, Goldbach R, et al. The NS3 protein of Rice hoja blanca virus suppresses RNA silencing in mammalian cells. J
Gen Virol, 2008, 89: 336-340

23 Xiong R, Wu J, Zhou Y, et al. Characterization and subcellular localization of an RNA silencing suppressor encoded by Rice stripe tenuivirus. Virology, 2009, 387: 29-40

24 Shi Y, Ryabov E V, Wezel R V, et al. Suppression of local RNA silencing is not sufficient to promote cell-to-cell movement of Turnip crinkle virus in Nicotiana benthamiana. Plant Signal Behav, 2009, 4: 15-22

25 Zhang L, Wang Z, Wang X, et al. Two virus-encoded RNA silencing suppressors, P14 of Beet necrotic yellow vein virus and S6 of Rice black streak dwarf virus. Chin Sci Bull, 2005, 50: 219-224

26 Lu R, Folimonov A, Shintaku M, et al. Three distinct suppressors of RNA silencing encoded by a $20-\mathrm{kb}$ viral RNA genome. Proc Natl Acad Sci USA, 2004, 101: 15742-15747

27 Gopal P, Kumar P P, Sinilal B, et al. Differential roles of C4 and beta $\mathrm{C} 1$ in mediating suppression of post-transcriptional gene silencing: Evidence for transactivation by the $\mathrm{C} 2$ of Bhendi yellow vein mosaic virus, a monopartite begomovirus. Virus Res, 2007, 123: 9-18

28 Omura T, Inoue H, Saito Y. Purification and some properties of Rice gall dwarf virus, a new Phytoreovirus. Phytopathology, 1982, 72: 1246-1249

29 Xie L H, Lin Q Y, Xie L Y, et al. Rice bunchy stunt virus: A new member of Phytoreovirus. J Fujian Agri Univ, 1996, 25: 312-319

30 Picton A, Potgieter C, Rey M E. Molecular analysis of six segments of Tobacco leaf enation virus, a novel Phytoreovirus from tobacco. Virus Genes, 2007, 35: 387-393

31 Katsar C S, Hunter W B, Sinisterra X H. Phytoreovirus-like sequences isolated from glassy-winged sharpshooter salivary glands (Hemiptera: Cicadellidae: Homalodisca vitripennis). Florida Entomol, 2007, 90: 196-203

32 Wei T, Ichiki-Uehara T, Miyazaki N, et al. Association of Rice gall $d$ warf virus with microtubules is necessary for viral release from cultured insect vector cells. J Virol, 2009, 83: 10830-10835

33 Francki R, Boccardo G. The plant reoviridae. In: The Reoviridae. New York: Plenum Press, 1983. 505-563

34 Ichimi K, Kikuchi A, Moriyasu Y, et al. Sequence analysis and GTP-binding ability of the minor core protein P5 of Rice gall dwarf virus. Jpn Agr Res Q, 2002, 36: 83-87

35 Moriyasu Y, Maruyama-Funatsuki W, Kikuchi A, et al. Molecular analysis of the genome segments S1, S4, S6, S7 and S12 of a Rice gall dwarf virus isolate from Thailand; completion of the genomic sequence. Arch Virol, 2007, 152: 1315-1322

36 Zhang H M, Yang J, Xin X, et al. Molecular characterization of the genome segments S4, S6 and S7 of Rice gall dwarf virus. Arch Virol, 2007a, 152: 1593-1602

37 Zhang H M, Xin X, Yang J, et al. Completion of the sequence of Rice gall dwarf virus from Guangxi, China. Arch Virol, 2008, 153: 17371741

38 Zhang H M, Yang J, Xin X, et al. Molecular characterization of the largest and smallest genome segments, S1 and S12, of Rice gall dwarf virus. Virus Genes, 2007b, 35: 815-823

39 Powers J G, Sit T L, Qu F, et al. A versatile assay for the identification of RNA silencing suppressors based on complementation of viral movement. Mol Plant Microbe In, 2008, 21: 879-890

40 Hamilton A J, Voinnet O, Chappell L, et al. Two classes of short interfering RNA in RNA silencing. EMBO J, 2002, 21: 4671-4679

41 Goto K, Kanazawa A, Kusaba M, et al. A simple and rapid method to detect plant siRNAs using nonradioactive probes. Plant Mol Biol Rep, 2003, 21: 51-58

42 Li W X, Ding S W. Viral suppressors of RNA silencing. Curr Opin Biotechnol, 2001, 12: 150-154

43 Chen J, Li W X, Xie D, et al. Viral virulence protein suppresses RNA silencing-mediated defense but upregulates the role of microRNA in host gene expression. Plant Cell, 2004, 16: 1302-1313

44 Voinnet O, Lederer C, Baulcombe D C. A viral movement protein prevents spread of the gene silencing signal in Nicotiana benthamiana. Cell, 2000, 103: 157-167

45 Pruss G, Ge X, Shi X M, et al. Plant viral synergism: The potyviral genome encodes a broad-range pathogenicity enhancer that transactivates replication of heterologous viruses. Plant Cell, 1997, 9: 859-868

46 Waterhouse P M, Helliwell C A. Exploring plant genomes by 
RNA-induced gene silencing. Nat Rev Genet, 2003, 4: 29-38

47 Cui X, Li G, Wang D, et al. A Begomovirus DNAbeta-encoded protein binds DNA, functions as a suppressor of RNA silencing, and targets the cell nucleus. J Virol, 2005, 79: 10764-10775

48 Li H W, Li W X, Ding S W. Induction and suppression of RNA silencing by an animal virus. Science, 2002, 296: 1319-1321

49 Lucy A P, Guo H S, Li W X, et al. Suppression of post-transcriptional gene silencing by a plant viral protein localized in the nucleus. EMBO J, 2000, 19: 1672-1680

50 Zhang X R, Yuan Y R, Pei Y, et al. Cucumber mosaic virus-encoded 2b suppressor inhibits Arabidopsis Argonaute1 cleavage activity to counter plant defense. Gene Dev, 2006, 20: 3255-3268

51 Wang Y Z, Tzfira T, Gaba V, et al. Functional analysis of the $\mathrm{Cu}$ cumber mosaic virus $2 \mathrm{~b}$ protein: Pathogenicity and nuclear localization. J Gen Virol, 2004, 85: 3135-3147

52 van Wezel R, Liu H T, Tien P, et al. Gene C2 of the monopartite geminivirus Tomato yellow leaf curl virus-China encodes a pathogenicity determinant that is localized in the nucleus. Mol Plant Microbe In, 2001, 14: 1125-1128

53 Dong X L, van Wezel R, Stanley J, et al. Functional characterization of the nuclear localization signal for a suppressor of posttranscriptional gene silencing. J Virol, 2003, 77: 7026-7033

Open Access This article is distributed under the terms of the Creative Commons Attribution License which permits any use, distribution, and reproduction in any medium, provided the original author(s) and source are credited. 